\title{
Sudden coronary death in Glasgow: the severity and distribution of chronic coronary atherosclerotic
} \section{stenoses}

\author{
M A EL FAWAL, * G A BERG, $\dagger$ D J WHEATLEY, $\dagger$ W A HARLAND* \\ From the ${ }^{\star}$ Department of Forensic Medicine and Science, University of Glasgow and the \\ †University Department of Cardiac Surgery, Royal Infirmary, Glasgow
}

SUMMARY A detailed analysis of the extent of coronary artery atherosclerosis was made in 92 white subjects ( 66 men and 26 women) who died suddenly from ischaemic heart disease. Stenoses resulting in loss of $\geqslant 75 \%$ of luminal cross sectional area (significant stenosis) were found in 90 subjects and these were more extensive in the proximal coronary tree than in the distal. Thirty nine per cent had triple vessel disease, $37 \%$ had double vessel disease, and $23 \%$ had single vessel disease. In addition one man had an isolated significant stenosis affecting the left main coronary artery. The frequency of significant stenoses in the left main coronary artery was greater in men than in women. The arteries that were least affected were the distal branches of the right coronary artery. A notable feature was the widespread nature of the coronary atherosclerosis: only 26 of the total of 1840 segments of coronary artery examined in the 92 victims could be described as having a normal intima ( $\leqslant 10 \%$ loss of the area within the internal elastic lamina).

The severity and the extent of advanced coronary atherosclerosis are recognised as important prognostic determinants in ischaemic heart disease, even when there are wide differences in age, race, geographical location, or other variables. ${ }^{1-3}$ Data relating coronary artery disease to clinical manifestations and risk factors are largely based on an assessment of left ventricular function and the extent and degree of coronary atherosclerosis. Therefore, accurate evaluation of the severity and extent of coronary atherosclerotic lesions is important to clinical cardiologists, epidemiologists, and pathologists investigating the disease.

We examined the extent, severity, and location of coronary atherosclerotic lesions in 130 cases of sudden death. The degree of stenosis was accurately estimated with a quantitative microscope to record the loss of luminal cross sectional area of the whole

Requests for reprints to Professor D J Wheatley, Department of Cardiac Surgery, Royal Infirmary, 10 Alexandra Parade, Glasgow G31 2ER.

Professor W A Harland died on 9 January 1985.

Accepted for publication 3 December 1986 epicardial tree after it had been divided into histological blocks taken at $3 \mathrm{~mm}$ intervals. Part of this study of sudden coronary death in Glasgow has already been reported. ${ }^{4}$

\section{Patients and methods}

We studied 130 white subjects under the age of 70 years (91 men and 39 women) who were submitted to medicolegal postmortem examination.

The coronary arteries were examined microscopically after postmortem angiography had been performed to determine the extent of coronary artery disease and the anatomy of the coronary arterial tree. The coronary arteries were dissected free from the heart, decalcified, and examined histologically. Blocks were taken at every $3 \mathrm{~mm}$ of the arteries and 0

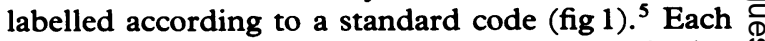
histological section was measured with a quantitative $\stackrel{\mathscr{P}}{?}$ microscope attached to a microcomputer. The degree of stenosis was estimated by calculating the area representing the original lumen enclosed by the internal elastic lamina and the area of the residual lumen. The stenosis was expressed as a percentage 0 loss of cross sectional area. The extent of athero- 


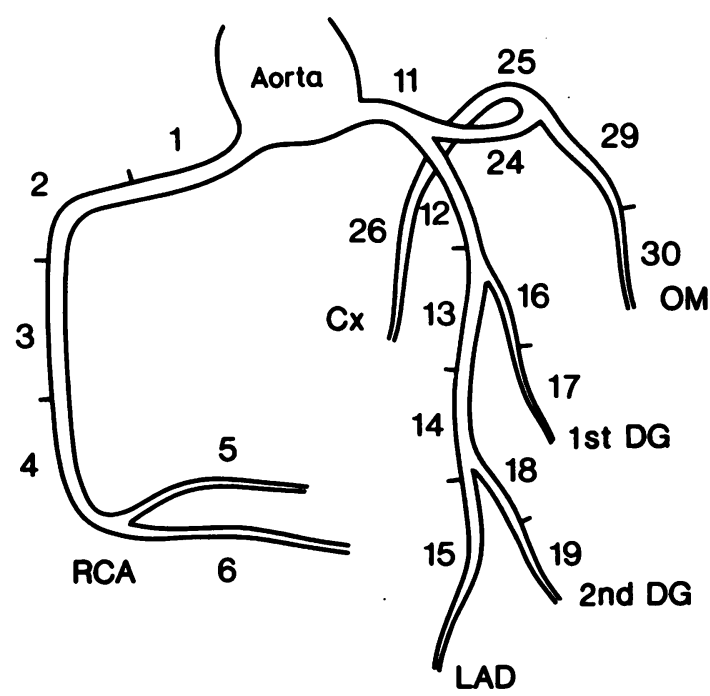

Fig 1 Division of coronary tree into segments. Right coronary artery $(R C A)$, segments 1-6; left main coronary artery, segment 11; left anterior descending artery (LAD), segments 12-15; first and second diagonal branches (1st DG and 2 nd $D G$ ), segments 16 and 17 and 18 and 19

respectively; circumflex artery $(C x)$, segments 24-26; obtuse marginal artery (OM), segments 29 and 30.

sclerotic narrowing in each segment of the coronary tree was measured and recorded as a percentage loss of luminal cross sectional area; those with stenoses $\geqslant 75 \%$ were considered to be "significantly" stenosed. Results are given both for the total number of significant stenoses in each segment and for the distance along the arteries to the segment with the first significant stenosis. Left main coronary artery disease, as well as single, double, or triple vessel disease, was noted. Subjects in whom there were limited areas of narrowing of one or two segments of the coronary tree with the remaining arteries relatively free of disease were noted. The positions of normal segments of artery (arbitrarily defined as loss of luminal cross sectional area $\leqslant 10 \%$ ) were noted.

\section{Results}

QUANTITATIVE ASSESSMENT OF SIGNIFICANT STENOSES

In 30 of the 130 subjects studied death was not caused by ischaemic heart disease. In eight subjects there was no identifiable cause of sudden death and, although coronary artery disease was present, there was no stenosis $\geqslant 75 \%$ and no acute coronary event. In these cases the cause of death was in doubt. The remaining 92 subjects showed evidence of severe atherosclerosis or acute coronary events with no other identifiable cause of sudden death. Significant stenoses (that is $\geqslant 75 \%$ loss of luminal cross sectional area) were found in 90 of the 92 cases of sudden ischaemic coronary death. When we compared the extent of stenoses in different parts of the coronary tree we found that certain segments showed a higher total number of significant stenoses than others (fig 2). The second and third $2 \mathrm{~cm}$ segments of the right coronary artery, the third and fourth $1.5 \mathrm{~cm}$ segments of the anterior descending coronary artery, and the $2 \mathrm{~cm}$ segment of the circumflex artery distal to its obtuse marginal branch had the highest number of significant stenoses.

\section{LOCATION OF SIGNIFICANT STENOSES}

Thirty five cases (39\%) had triple vessel disease. Twenty five of these showed stenoses of $\geqslant 80 \%$ in the three vessels, and in four cases the stenosis in each of the three vessels was $\geqslant 90 \%$. Thirty three subjects $(37 \%)$ had significant double vessel disease-four with an isolated stenosis in each affected vessel. Twenty one subjects $(23 \%)$ had single vessel disease-five with one isolated stenosis and five having two discrete stenoses. One subject had a significant high grade stenosis in his left main coronary artery only.

The total number of significant lesions (fig 2) was greater in the proximal coronary tree than in the distal (312 and 197 stenoses respectively $(p<0.01)$ ).

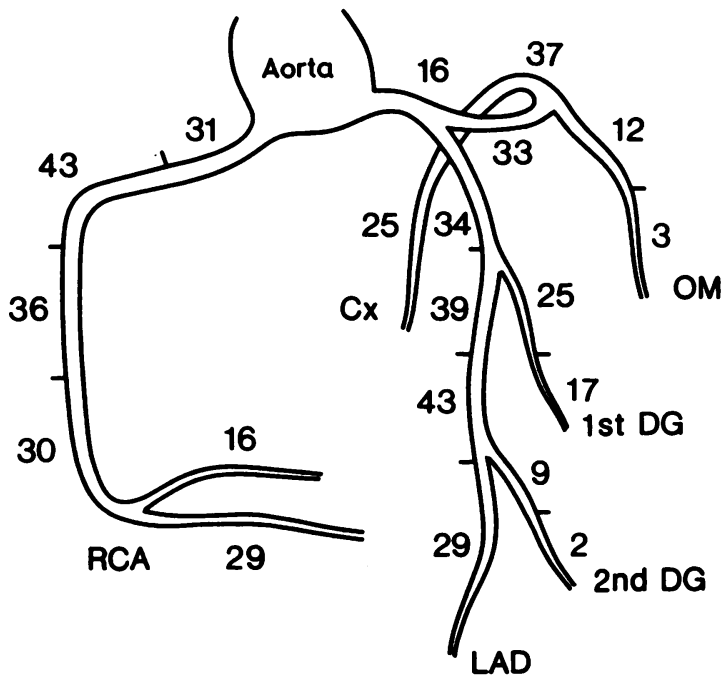

Fig 2 Total number of significant stenoses in each segment of the coronary tree in 92 subjects (configuration as fig 1). $R C A$, right coronary artery; $L A D$, left anterior descending artery; 1st $D G$, first diagonal branch of $L A D ; 2 n d D G$, second diagonal branch of $L A D ; C x$, circumflex artery; $O M$, obtuse marginal artery. 
422

Proximal segments for the purpose of this study were defined as the ten segments $1,2,3,11,12,13,14,16$, 24 , and 29 , and the ten distal segments were defined as $4,5,6,15,17,18,19,25,26$, and 30 (fig 1). The increased severity of proximal disease was reflected in all three major coronary arteries. In 17 of the 92 cases, however, the distal parts of one or more of the main branches were more severely narrowed than the proximal parts. Twelve of these 17 cases had single distal lesions (that is one distal lesion in one of the major branches, with the rest of the vessels being relatively disease free). Four subjects had two vessels with distal lesions and one had three vessels with distal lesions.

The most frequent location for the first significant lesion encountered (that is the most proximal) along the coronary tree was the first segment of all three main branches (fig 3).

\section{DISEASE OF THE LEFT MAIN CORONARY}

ARTERY

The total number of significant lesions in the left main coronary artery was significantly greater in men than women (fig $4(p<0.02)$ ). There was no difference in the frequency of disease between those

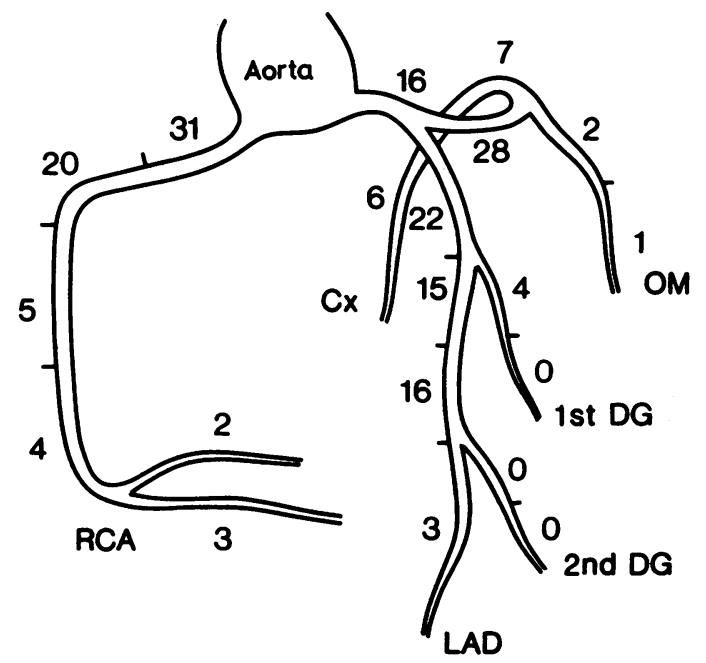

Fig 3 Location of the most proximal significant stenosis in 92 subjects. $R C A$, right coronary artery; $L A D$, left anterior descending artery; 1 st DG, first diagonal branch of $L A D ; 2 n d D G$, second diagonal branch of $L A D ; C x$, circumflex artery; OM, obtuse marginal artery.

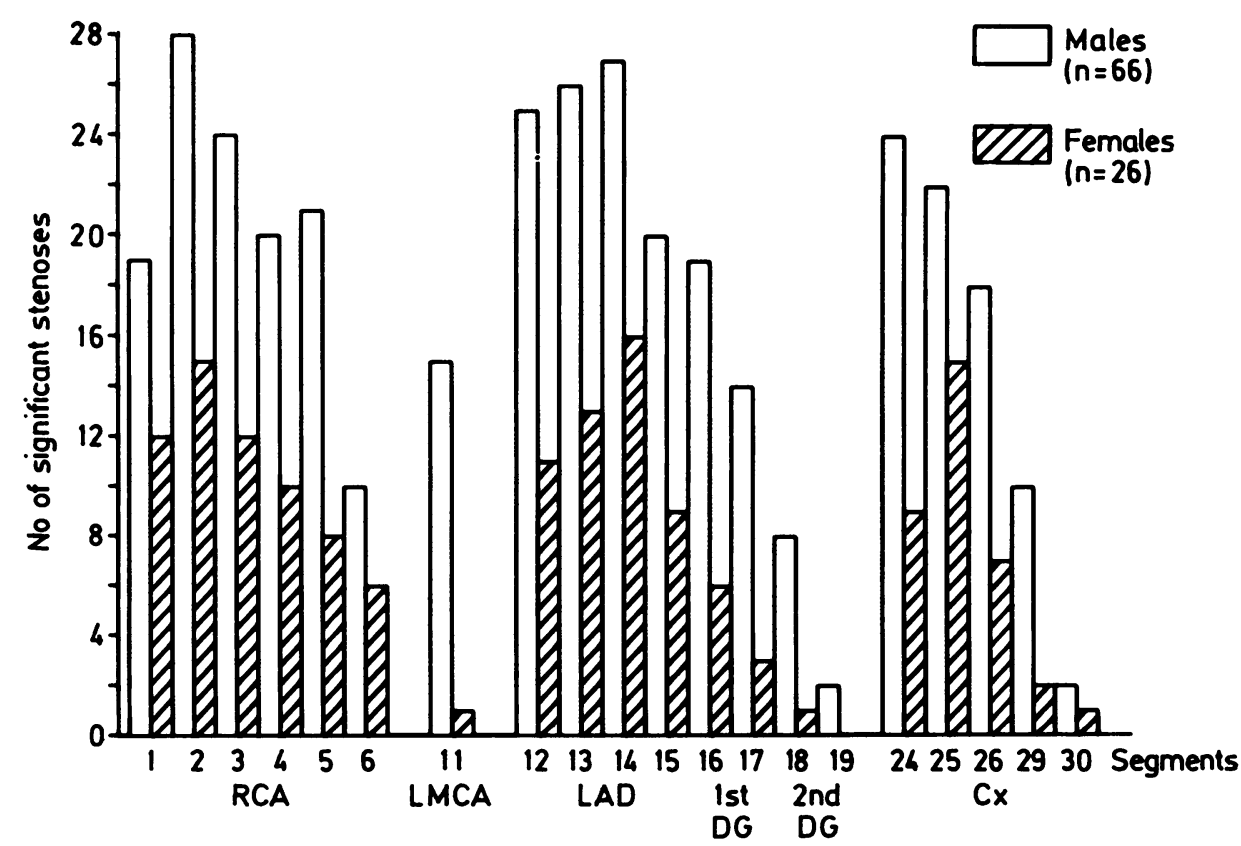

Fig 4 Histogram of the numbers of significant stenoses in each segment for men and women. Numbers indicate segments as shown in fig 1. RCA, right coronary artery; LMCA, left main coronary artery; $L A D$, left anterior descending artery; 1 st $D G$, first diagonal branch of $L A D$; 2nd $D G$, second diagonal branch of $L A D ; C x$, circumflex artery. 


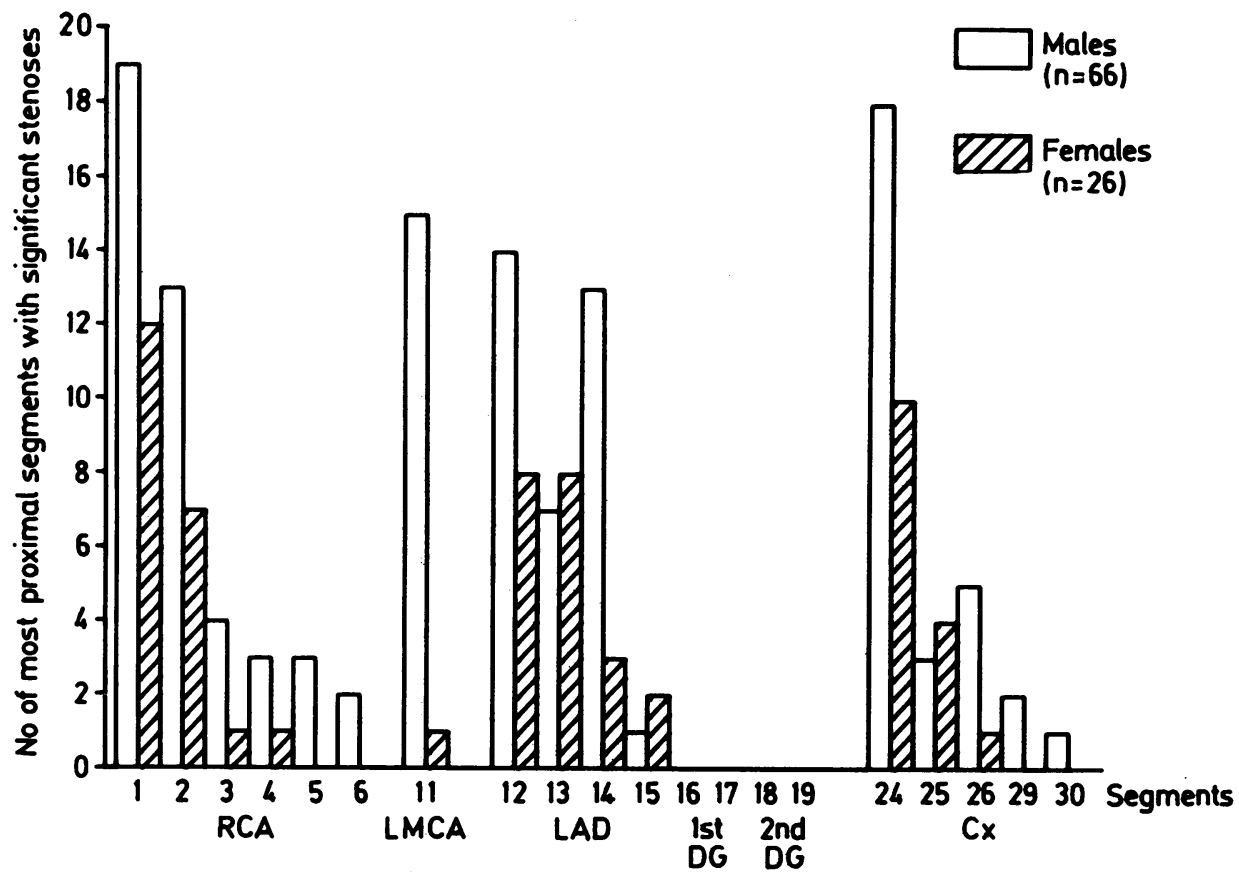

Fig 5 Histogram of the number of the most proximal segments with significant stenoses in men and women. Numbers indicate segments as shown in fig $1 . R C A$, right coronary artery; LMCA, left main coronary artery; $L A D$, left anterior descending artery; 1st $D G$, first diagonal branch of $L A D ; 2 n d D G$, second diagonal branch of $L A D ; C x$, circumflex artery.

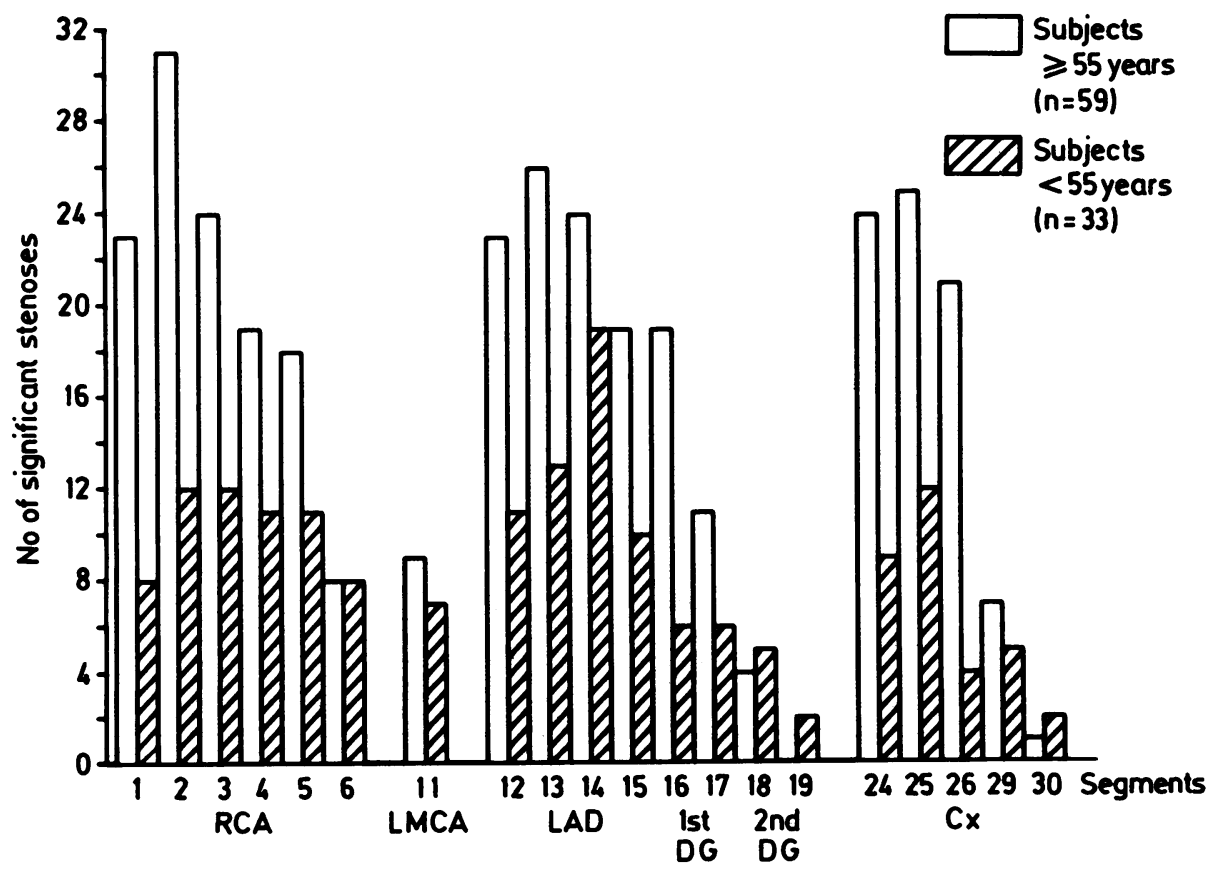

Fig 6 Histogram of the numbers of significant stenoses in each segment for groups aged less than 55 years and more than 55 years. Numbers indicate segments as shown in fig $1 . R C A$, right coronary artery; LMCA, left main coronary artery; LAD, left anterior descending artery; 1 st $D G$, first diagonal branch of $L A D ; 2 n d D G$, second diagonal branch of $L A D ; C x$, circumflex artery. 
aged $\geqslant 55$ and those aged $<55$. Isolated severe stenosis of the left main coronary artery occurred in only one case and there were no stenoses $>90 \%$.

\section{SEX AND AGE DISTRIBUTION}

There was no difference between the total number of significant lesions (or the distance to the first significant stenosis) for men and women (fig 5). Similarly, there was no difference for those aged $<55$ years and those aged $\geqslant 55$ years (fig 6 ).

\section{NORMAL SEGMENTS}

Segments with minimal atheroma (loss of cross sectional area $<25 \%$ ) (fig 7) were more common in the distal coronary tree than in the proximal ( $136 \mathrm{com}$ pared with 50 segments respectively $(p<0.01)$ ). There were more normal segments in the right coronary artery than in the anterior descending or circumflex arteries. Only 26 segments had stenoses causing $<10 \%$ loss of cross sectional area.

\section{OLD LESIONS}

Old lesions, represented by recanalisation, were found in 27 subjects; 24 of them had additional recent coronary lesions. Figure 8 shows the distribution of these old lesions, which were more common in the distal or smaller vessels. No old lesions were found in the proximal anterior descending artery, but more old lesions than acute lesions were found in the diagonal branches of this vessel.

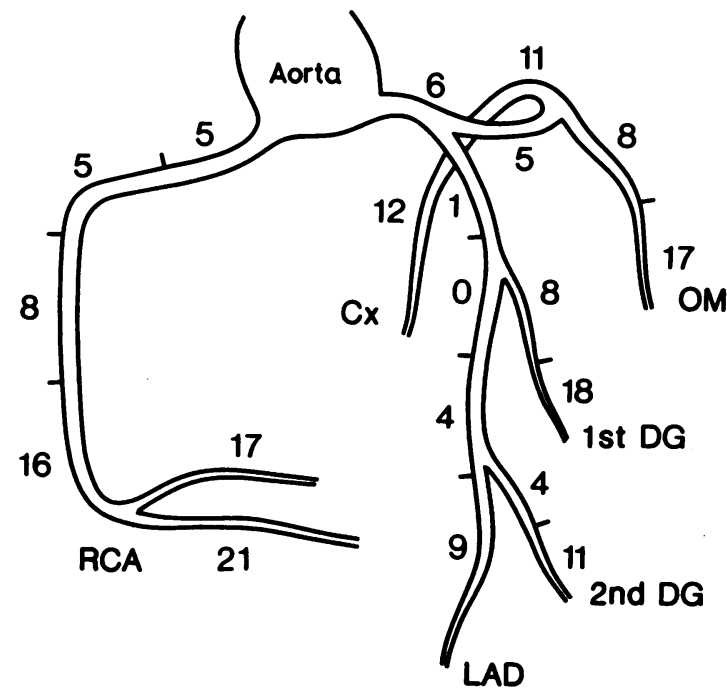

Fig 7 Location of segments with loss of cross sectional area $<25 \%$. Results from 92 subjects.

\section{DEATHS FROM CAUSES OTHER THAN ISCHAEMIC HEART DISEASE}

Four of the 30 victims of sudden death with a cause other than ischaemic heart disease had significant stenoses. The range of loss of luminal cross sectional area was $7 \%-92 \%$ and the mean maximum loss of luminal area was $41.5 \%$. Normal segments (loss of

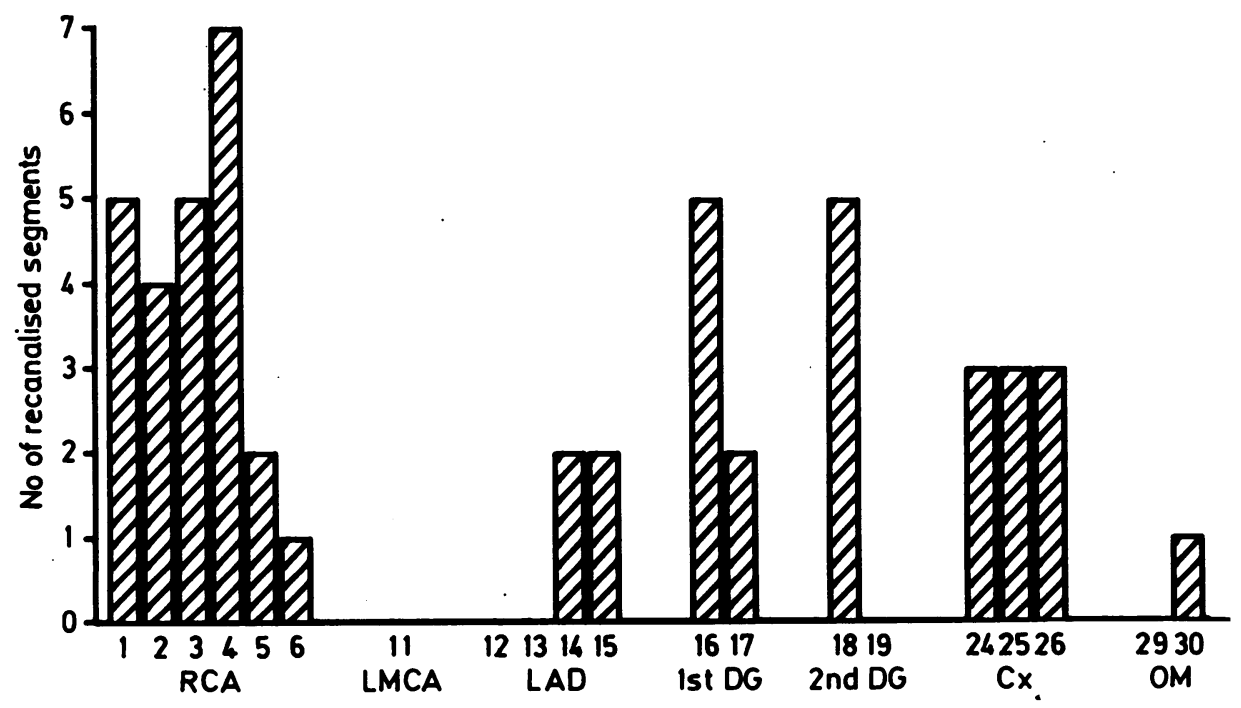

Fig 8 Distribution of recanalised segments of coronary artery in 27 cases (numbers indicate segments as shown in fig 1). RCA, right coronary artery; LMCA, left main coronary artery; $L A D$, left anterior descending artery; 1st $D G$, first diagonal branch of $L A D ; 2$ nd $D G$, second diagonal branch of LAD;Cx, circumflex artery; OM, obtuse marginal artery. 
luminal cross sectional area of $<10 \%$ ) occurred in 56 out of the 600 segments of coronary arteries examined in these 30 subjects.

\section{Discussion}

It is well recognised that there are considerable age, sex, racial, and geographic variations in the prevalence of atherosclerosis. ${ }^{46} \mathrm{~A}$ necropsy is not always performed on every patient with ischaemic heart disease who dies suddenly. For this reason the composition of groups of cases of sudden coronary death can be highly variable.

The most important measurement in the coronary tree of patients with coronary artery disease is the degree of stenosis. ${ }^{7}$ The degree of coronary narrowing can be estimated with considerable precision ${ }^{8}$; however, an accurate evaluation of atherosclerotic lesions is only possible at necropsy. Techniques for the evaluation of the amount of coronary artery stenosis vary. In practice, pathologists have tended to use individual methods that render comparison rather difficult. Tracing the circumference of the residual lumen and the internal elastic lamina with a quantitative microscope is an accurate technique that can be reproduced in different populations.

A further difficulty arises in defining what is meant by a "severe" or "significant" stenosis, particularly in the context of sudden death. Many consider a stenosis producing $75 \%$ loss of the luminal cross sectional area as a significant factor in sudden coronary death..$^{910}$ The problem is further complicated by the question of whether the state of the arterial lumen at necropsy is a true index of the calibre during life. It is possible that factors such as artefacts produced by postmortem contraction and fixation may affect lumen size and shape. It has been suggested that when an artery is fixed under pressure similar to normal arterial blood pressure, raised plaques tend to recede into small concavities in the media. $^{11}$

In this study the coronary tree was examined angiographically at necropsy to help in determining the cause of death and to localise areas of interest for sectioning. In our experience changing the injection pressure did not materially alter the degree of existing stenosis, provided that adequate filling of distal epicardial vessels was achieved. On the other hand, failure to fill the arteries completely with injection medium can lead to the collapse of the histological preparations and the eventual overestimation of the degree of narrowing by planimetry.

This study shows that in the majority of cases of sudden coronary death the proximal segments of the main branches of the coronary tree are more severely affected than the distal segments. Such findings may accord with the suggested importance of the role of haemodynamic factors in plaque formation.

Atherosclerotic lesions were widespread in all the subjects in this study (even those not dying of ischaemic heart disease). There was disease in most segments examined. Only 26 of the 1840 segments examined from the 92 subjects in whom death from ischaemic heart disease was confirmed had a loss of coronary artery cross sectional area $\leqslant 10 \%$. Only 56 of the 600 segments from the 30 subjects who died of causes other than ischaemic heart disease had a loss of cross sectional area $\leqslant 10 \%$. Many of these histologically diseased segments had an angiographically normal appearance.

This study has shown that a considerable proportion of subjects with ischaemic heart disease who die suddenly have only single vessel disease and this group, particularly, might be considered to have a good prognosis if seen in life. All cases warrant full resuscitative efforts. This study and our previous on $\mathrm{e}^{4}$ provide further evidence that the same pathological processes underlie sudden death from ischaemic heart disease and unstable angina or evolving myocardial infarction. ${ }^{12}$

This work was supported by a grant from the Scottish Hospital Endowments Research Trust.

\section{References}

1 Guzman MA, McMahan CA, McGill HC, et al. Selected methodologic aspects of the International Atherosclerosis Project. Lab Invest 1968;18:479-97.

2 Deupree RH, Fields RI, McMahan CA, Strong JP. Atherosclerotic lesions and coronary heart disease. Key relationships in necropsied cases. Lab Invest 1973;28:252-62.

3 Takaro $\mathrm{T}$ and the participants in the VA Co-operative Study of surgery for coronary arterial occlusive disease. Results of a randomised study of medical and surgical management of angina pectoris. World $J$ Surg 1978;2:797-809.

4 El Fawal MA, Berg GA, Wheatley DJ, Harland WA. Sudden coronary death in Glasgow: nature and frequency of acute coronary lesions. $\mathrm{Br}$ Heart $\mathrm{J}$ 1987;57:329-35.

5 Brandt PW, Partridge JB, Wattie WJ. Coronary arteriography: method of presentation of the arteriogram report and a scoring system. Clin Radiol 1977; 28:361-5.

6 Sacks MI. Aortic and coronary atherosclerosis in the three racial groups in Cape Town. Circulation 1960;22:96-109.

7 Crawford T. Pathology of ischaemic heart disease. London: Butterworth, 1977:26.

8 Wijns W, Surruys PW, Reiber JH, et al. Quantitative angiography of the left anterior descending coronary 
artery: correlations with pressure gradient and results of exercise thallium scintigraphy. Circulation 1985;71:273-9.

9 Davies MJ, Thomas A. Thrombosis and acute coronary artery lesions in sudden cardiac ischaemic death. $N$ Engl J Med 1984;310:1137-40.

10 Warnes CA, Roberts WC. Sudden coronary death: comparison of patients with, to those without, coronary thrombus at necropsy. Am J Cardiol 1984;
54:1206-11.

11 Crawford $T$, Levene CI. Incorporation of fibrin in the aortic intima. J Path. Bact 1952;64:523-8.

12 Falk E. Unstable angina with fatal outcome: dynamic coronary thrombosis leading to infarction and/or sudden death. Autopsy evidence of recurrent mural thrombosis with peripheral embolisation culminating in total vascular occlusion. Circulation 1985;71: 699-708. 